\title{
Equilibrium and Kinetic Studies of Acid Dye Adsorption on Palm Oil Empty Fruit Bunch
}

\author{
Norini Tahir ${ }^{1 *}$ and Zaiton Abdul Majid ${ }^{2}$ \\ ${ }^{1}$ School of Science and Technology, UMS, 88400 Kota Kinabalu, Sabah, Malaysia \\ ${ }^{2}$ Department of Chemistry, Faculty of Science, UTM, 81310 UTM Skudai, Johor, Malaysia \\ Received 10 December 2012, Revised 30 March 2013, Accepted 22 April 2013, Available online 4 May 2013
}

\begin{abstract}
Palm oil empty fruit bunch (POEFB), an agricultural waste, used as low-cost adsorbent for removal Acid Orange 51 (AO51) dye from aqueous solutions was studied. Batch mode experiments were carried out at room temperature $(28 \pm 2){ }^{\circ} \mathrm{C}$ to study the effects of contact time and initial dye concentrations $(10-200 \mathrm{mg} / \mathrm{L})$. The equilibrium adsorption data of AO51 dye on empty fruit bunch were analyzed by three isotherms, namely the Langmuir isotherm, Freundlich isotherm and Temkin isotherm model. The result indicated that the equilibrium sorption fitted well with the Freundlich isotherm, displaying higher regression coefficient, $R^{2}$ value. The monolayer adsorption capacity of POEFB was found to be $166.67 \mathrm{mg} / \mathrm{g}$. The kinetic data obtained at different concentrations have been analyzed using the pseudo-first-order, pseudo-second-order and intraparticle diffusion models. It was shown that pseudo-second-order kinetic model could best describe the adsorption kinetics.
\end{abstract}

| Palm oil empty fruit bunch | Low-cost adsorbent | Acid Orange 51 | Adsorption isotherm | Kinetics |

(B 2013IbnuSina Institute. All rights reserved. http://dx.doi.org/10.11113/mjfas.v9n3.100

\section{INTRODUCTION}

The presence of dyes in effluents is a major concern due to their adverse effects to many forms of life. The discharge of dyes in the environment is a matter of concern for both toxicological and esthetical reasons. Industries such as textile, leather, paper and plastics use dyes in order to colour their products and also consume substantial volumes of water. As a result, they generate a considerable amount of coloured wastewater. It is estimated that more than 100,000 commercially available dyes with over $7 \times 10^{5}$ tonnes of dyestuff produced annually and from 40,000 to 50,000 tonnes of dye are discharged to surface water every year [1]. The presence of even very small amounts of dyes in water (less than $1 \mathrm{mg} / \mathrm{L}$ for some dyes) is highly visible and undesirable [2,3]. Due to large-scale production and extensive application, synthetic dyes can cause considerable environmental pollution and are serious health-risk factors [4]. Acid dyes are widely used in the textile industry. These water soluble anionic dyes are mostly used with certain fibre types such as polyamide, wool, silk, modified acrylic, and polypropylene fibres, as well as blends of the aforementioned fibres with other fibres such as cotton, rayon, polyester and regular acrylic. Acid dyes are also used for dyeing other materials like leather and paper. However, the majority of these dyes are synthetic in nature and are usually composed of aromatic rings in their structure, whichmakes them carcinogenic and mutagenic, inert and non- biodegradable when discharged into waste streams.
The treatment of wastewater has long been a major concern of the textile industry. During the past three decades, several physical, chemical and biological decolourization methods have been reported. Adsorption is a well known equilibrium separation process and an effective method for water decontamination applications. Recently, numerous approaches have been studied for the development of cheaper and effective adsorbents. Adsorption on activated carbon has been shown to be very effective for removal of dyes and other pollutants form aqueous solutions. However, commercially available activated carbon is still considered expensive due to the use of non-renewable and relatively expensive starting material such as coal, which is unjustified in pollution control applications [5]. Various extensive researches has been undertaken recently to develop alternative and economic adsorbents. A number of non-conventional sorbents has been reported in the literature for their capacity to remove acid dye from aqueous solutions, such as coal [6], chitosan [7], water hyacinth roots [8], sludge [9] and modified starch [10].

In this study, we attempt to utilize palm oil empty fruit bunch (POEFB), an agricultural solid waste, as an alternative low-cost sorbent in the removal of acid orange 51 dye from aqueous solutions. Palm oil empty fruit bunches (POEFB) is one of the major solid wastes from oil palm industry in Malaysia besides the trunk and shell. 30 million tonnes of palm oil wastes are produced annually which include trunks, fronds, leaves and empty fruit 
bunches [11]. For each bunch of the fresh palm fruit, approximately $21 \%$ of palm oil, $6-7 \%$ of palm kernels, 14 $15 \%$ of palm fibres, $6-7 \%$ of palm shells and $23 \%$ of empty fruit bunches can be obtained [12]. Just in Malaysia, more than two million tonnes (dry weight) of extracted palm oil fibre are estimated to be generated annually [13]. POEFB has traditionally been burnt and their ash recycled into the plantation as fertilizer. However, due to the pollution problem, incineration of POEFB has been discouraged. In the last few years, several research teams began to regard the POEFB and other residues from the palm oil industry as sources of energy or converting it into value added product. Until today, a number of utilization techniques have been developed so as to exploit in a rational way these biomass residues. These techniques include the production of fibreboard, pulp, composites and also as an adsorbent for wastewater treatment [11]. Researchers have worked on palm oil empty fruit bunch as an effectiveness adsorbent for dyes removal from wastewater such as anionic dyes [14], Malachite Green [15], Methyl Red [16] and Methylene Blue [17]. However, no studies have been reported on the use of POEFB for the removal of acid dye from wastewater. Thus, the objective of this study is to investigate the potential of POEFB as a low-cost adsorbent for removal of acid dye, acid orange 51, from aqueous solutions.

\section{EXPERIMENTAL}

\subsection{Adsorbate}

Acid dye, Acid Orange 51 (AO51) was purchased from Sigma-Aldrich (Malaysia) and used without further purification. The maximum absorption wavelength of this dye is $455 \mathrm{~nm}$ and the chemical structure is shown in Figure 1. Stock solution of $250 \mathrm{ppm}$ of dye was prepared by dissolving an accurately weighed quantity of dye, in distilled water. Experimental solution of the desired concentration was obtained by successive dilutions.

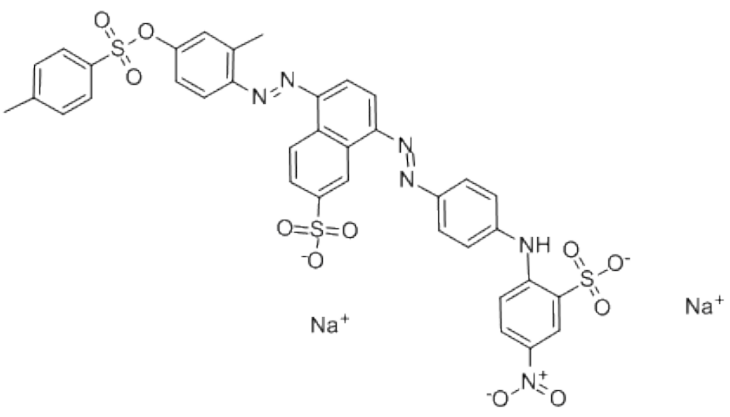

Fig. 1 Chemical structure of Acid Orange 51

\subsection{Preparation and Characterization of Adsorbent}

The palm oil empty fruit bunch waste was collected from the palm oil estate in Kulai, Johor. The collected material was left to dry naturally out site the laboratory. The dried material was firstly chopped to pieces approximately 1 in. ${ }^{3}$ in size, washed repeatedly with boiled water till the washed water is clear from any colour and finally washed with distilled water and oven dried at $70^{\circ} \mathrm{C}$ for 48 hours to constant weight. The prepared samples was then ground and sieved to obtain particle size of $\sim 75 \mu \mathrm{m}$. The dried samples were stored in plastic bottle for further use. No other chemical or physical treatments were used prior to adsorption experiments.

Chemical characterizations of surface functional groups on the adsorbent prepared were carried out using a Fourier Transform Infrared Spectroscope (Spectrum One FTIR, Perkin Elmer). The spectra were recorded from 4000 to $400 \mathrm{~cm}^{-1}$. In addition, Scanning Electron Microscopy (SEM) analysis was carried out to study the surface morphology of the prepared adsorbent.

\subsection{Adsorption Studies}

The batch adsorption studies were conducted at room temperature ( $28 \pm 2{ }^{\circ} \mathrm{C}$ ) to obtain the adsorption rates and equilibrium data. The experiments were carried out by adding a fixed amount $(0.1 \mathrm{~g})$ of the prepared sample into a set of $100 \mathrm{~mL}$ Erlenmeyer flasks containing $25 \mathrm{~mL}$ of different initial concentrations (10, 25, 50, 100 and 200 $\mathrm{mg} / \mathrm{L}$ ) of acid orange 51 dye solutions. The flasks were sealed with parafilm and then agitated on a rotary orbital shaker at 160 rpm for 3 hours until equilibrium was reached. Aqueous samples were taken from the solutions and the concentrations were analyzed. All samples were filtered with filter paper to separate the adsorbents from the aqueous solution. The concentrations of AO51 dye in the supernatant solutions before and after adsorption were determined at maximum wavelength $\left(\lambda_{\max }\right) 455 \mathrm{~nm}$ using a UV-Vis spectrometer (Perkin Elmer Lambda 25). The amount of adsorption at equilibrium, $q_{e}(\mathrm{mg} / \mathrm{g})$, was calculated by

$$
q_{e}=\frac{\left(C_{0}-C_{e}\right) V}{W}
$$

where $C_{0}$ and $C_{e}(\mathrm{mg} / \mathrm{L})$ are the liquid-phase concentrations of dye at initial and equilibrium, respectively. $V$ is the volume of the solution (L) and $W$ is the mass of dry adsorbent used (g).

Kinetic studies of adsorption were also carried out at various concentrations of the AO51 dye solutions wherein the extent of adsorption was investigated as a function of time. The amount of adsorption at time $t, q_{t}(\mathrm{mg} / \mathrm{g})$, was calculated by

$$
q_{t}=\frac{\left(C_{0}-C_{t}\right) V}{W}
$$




\section{RESULTS \& DISCUSSION}

\subsection{Characterization of the Adsorbent}

The FTIR spectrum of POEFB given in Figure 2 shows peak at $3393.57 \mathrm{~cm}^{-1}$ (O-H stretching), $2919.88 \mathrm{~cm}^{-1}$ (C-H stretching), $2367.12 \mathrm{~cm}^{-1}(\mathrm{C} \equiv \mathrm{O}), 1717.29 \mathrm{~cm}^{-1}(\mathrm{C}=\mathrm{O})$, $1638.29 \mathrm{~cm}^{-1}$ (C=C aromatic), $1250.09 \mathrm{~cm}^{-1}(\mathrm{C}-\mathrm{OH})$ and $1053.09 \mathrm{~cm}^{-1}(\mathrm{C}-\mathrm{O})$. It is clear that the adsorbent displays a number of absorption peaks, reflecting the complex nature of the adsorbent.

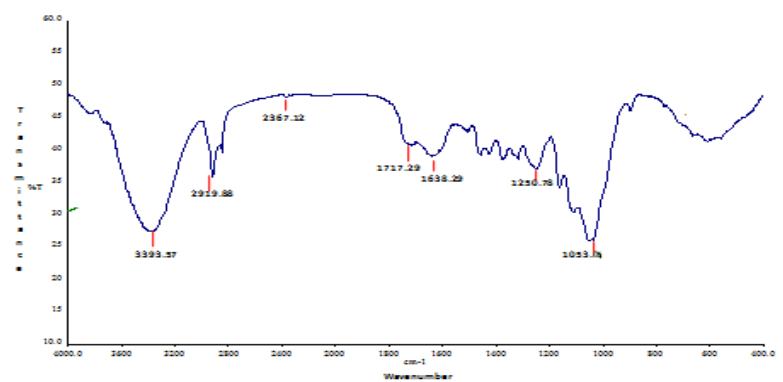

Fig. 2 FTIR spectra of POEFB

Figure 3 shows the SEM micrograph of POEFB fibre. The micrograph shows small particles of fibre are scattered randomly all over the fibre surface. The POEFB exhibits a rough and uneven surface morphology.

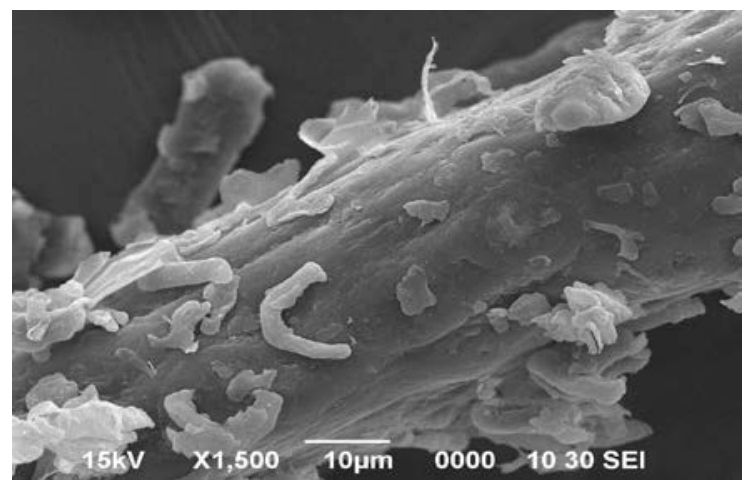

Fig. 3 SEM image of POEFB

\subsection{Effect of Initial Dye Concentration andContact Time on Dye Adsorption}

In order to study the effect of the initial concentration of AO51 in the solutions on the rate of adsorption of POEFB, the experiments were carried out at a fixed adsorbent dosage of $0.1 \mathrm{~g}$ and at different initial dye concentrations $(10,25,50,100$ and $200 \mathrm{mg} / \mathrm{L})$ for differenttime intervals in the range of 10-200 minutes.
Figure 4 shows the effect of the initial dye concentration on the adsorption. The decrease in residual concentration of AO51indicates an increase in the amount of dye adsorbed as afunction of time. As the initial concentrations increases from $10-200 \mathrm{mg} / \mathrm{L}$, the adsorption removal also increases from 1.25 to $32.64 \mathrm{mg} / \mathrm{g}$. It is also shown in Figure 4, after a lapse of approximately $20 \mathrm{~min}$, a gradual approach to the limiting adsorption was observed. For AO51 solutions with initial concentrations of $10-50 \mathrm{mg} / \mathrm{L}$, the contact time needed to reach equilibrium at $80 \mathrm{~min}$ while for $\mathrm{AO51}$ solutions with initial concentration of $100-200 \mathrm{mg} / \mathrm{L}$, equilibrium time of $180 \mathrm{~min}$ was required. However, the experimental data were measured at $200 \mathrm{~min}$ to make sure full equilibrium was attained.

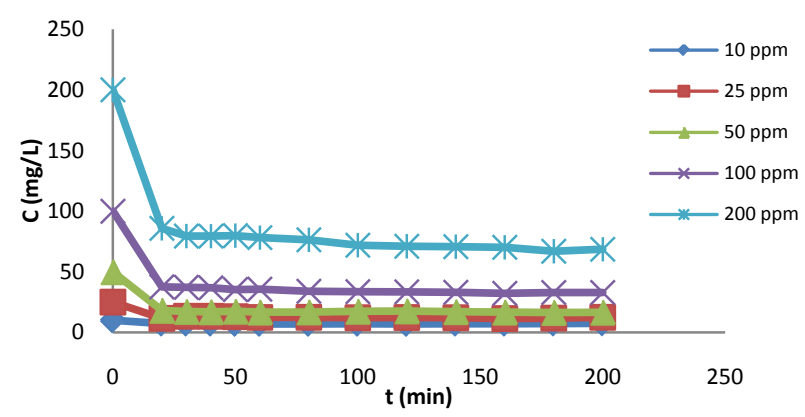

Fig. 4 Effect of initial concentration and contact time on A051 adsorption

\subsection{Equilibrium Isotherms}

The adsorption isotherm indicates how the adsorption molecules are distributed between the liquid phase and the solid phase when the adsorption process reaches an equilibrium state. Adsorption isotherm is basically important in describing how solutes interact with adsorbents, and is critical in optimizing the use of adsorbents [18]. The data obtained from the equilibrium studies were analyzed according to Langmuir, Freundlich and Temkin adsorption isotherms. The applicability of the isotherm models to the adsorption study done was compared by judging the correlation coefficients, $R^{2}$ values.

The Langmuir adsorption model is based on the assumption that maximum adsorption corresponds to a saturated monolayer of solute molecules on the adsorption surface, with no lateral interaction between the sorbed molecules [19]. The expression of the Langmuir model is given by Eq. (3)

$$
q_{e}=\frac{q_{\max } a C_{e}}{\left(1+a C_{e}\right)}
$$

where $q_{e}(\mathrm{mg} / \mathrm{g})$ and $C_{e}(\mathrm{mg} / \mathrm{L})$ are the amount of adsorbed dye per unit mass of sorbent and equilibrium concentration, respectively. $q_{\max }$ is the maximum amount of the dye per unit mass of sorbent to form a complete monolayer on the 
surface bound at high $C_{e}$ and a is a constant related to the affinity of the binding site. The Langmuir equation can be described by the linearized form:

$$
\frac{1}{q_{e}}=\frac{1}{q_{\max }}+\frac{1}{a q_{\max } C_{e}}
$$

The linear plots of specific adsorption $\left(C_{e} / q_{e}\right)$ against the equilibrium concentration $\left(C_{e}\right)$ for dye ions adsorption onto POEFB fibre is shown in Figure 5. The isotherm constants, $K_{L}$, and equilibrium monolayer capacities, $q_{\max }$, were determined from the slope and intercept of the plots and are presented in Table 1. Analyses of the experimental data show that the Langmuir model is not an appropriate prediction of the isothermal profiles for the sorption of the investigated acid dye on POEFB fibre. The low $R^{2}$ value (Table 1) shows that the adsorption isotherms do not fit the Langmuir isotherm. The inability of the Langmuir model to represent the experimental data could have been due to the fact that this isotherm does not take into account adsorbateadsorbate interactions.

The essential characteristics of the Langmuir isotherm can be expressed in terms of a dimensionless constant separation factor $R_{L}$ that is given in Eq. (5)

$$
R_{L}=\frac{1}{1+a C_{0}}
$$

where $C_{0}$ is the highest initial concentration of adsorbate (mg/L) and $a$ is Langmuir constant. The value of $R_{L}$ indicates the shape of the isotherm to be either unfavourable $\left(R_{L}>1\right)$, linear $\left(R_{L}=1\right)$, favourable $\left(0<R_{L}<1\right)$, or irreversible $\left(R_{L}=0\right)$. The value of $R_{L}$ was found to be 0.63 , thus confirming that POEFB fibre favours the adsorption of acid dye under the condition used in this study.

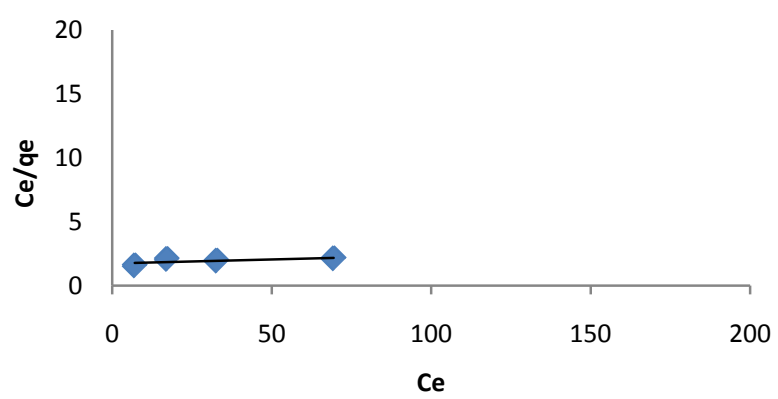

Fig. 5 Langmuir isotherm for AO51 dye adsorption on POEFB

The Freundlich isotherm is used for non-ideal adsorption on heterogeneous surfaces. The heterogeneity arises from the presence of different functional groups on the surface, and the various adsorbent-adsorbate interactions [20]. The Freundlich equation is expressed as:

$$
\log q_{e}=\log K_{F}+\frac{1}{n} \log C_{e}
$$

where $K_{F}$ and $n$ are Freundlich constants with $K_{\mathrm{F}}$ is the adsorption capacity of the sorbent and $n$ giving an indication of how favourable the adsorption process. The magnitude of the exponent, $1 / n$, gives an indication of the favourability of adsorption. Values of $n>1$ represent favourable adsorption condition. Theconstants, $K_{F}$ and $n$, were determined from a graph of $\log q_{e}$ against $\log C_{e}$ (Figure 6).

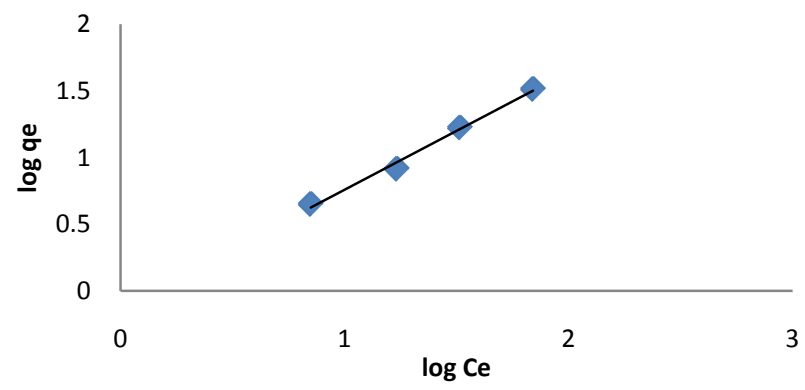

Fig. 6 Freundlich isotherm for AO51 dye adsorption on POEFB

The Freundlich isotherm parameters and the linear regression coefficients are also shown in Table 1. It is clear from the Table 1 that the values of the Freundlich heterogeneity factor $1 / \mathrm{n}$ is smaller than $1 ; 1 / \mathrm{n}<1$, represents favourable adsorption condition. The $R^{2}$ value result shows that Freundlich isotherm fitted the equilibrium data for adsorption of AO51 dye on POEFB fibre. As a result, it can be said that the overall adsorption of AO51 dye in this study obeys the Freundlich isotherm in which adsorption is based on heterogeneous surface.

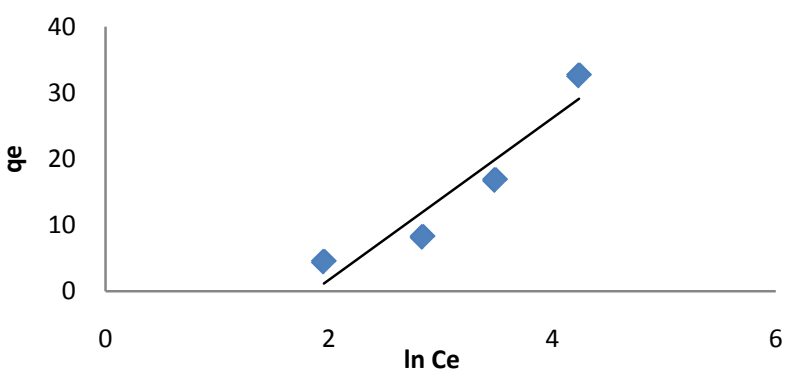

Fig. 7 Temkin isotherm for AO51 dye adsorption on POEFB

The Temkin isotherm equation assumes that the heat of adsorption of all the molecules in the layer decreases linearly with coverage due to adsorbate-adsorbate interactions, and that the adsorption is characterized by a uniform distribution of the binding energies, up to some maximum binding energy [21]. The Temkin isotherm has been generally applied in the following form:

$$
q_{e}=\left(\frac{R T}{b_{T}}\right) \operatorname{In}\left(A C_{e}\right)
$$


Table 1 Langmuir, Freundlich and Temkin isotherms constants, correlation coefficients and separation factor for adsorption of AO51 on POEFB

\begin{tabular}{lc}
\hline Isotherms & Parameters \\
\hline Langmuir & \\
$q_{\max }(\mathrm{mg} / \mathrm{g})$ & 166.67 \\
$K_{L}(\mathrm{l} / \mathrm{mg})$ & 0.50 \\
$R^{2}$ & 0.47 \\
$R_{L}$ & 0.63 \\
Freundlich & \\
$K_{F}\left(\mathrm{mg} / \mathrm{g}(\mathrm{l} / \mathrm{mg})^{1 / \mathrm{n}}\right)$ & 0.75 \\
$1 / n$ & 0.88 \\
$R^{2}$ & 0.99 \\
Temkin & \\
$A(1 / \mathrm{g})$ & 0.16 \\
$B$ & 12.22 \\
$R^{2}$ & 0.90 \\
\hline
\end{tabular}

and can be linearized as:

$$
q_{e}=\frac{R T}{b} \operatorname{In} A+\frac{R T}{b} \operatorname{In} C_{e}
$$

whereb is the Temkin constant related to heat of sorption $(\mathrm{J} / \mathrm{mol}), A$ is the Temkin isotherm constant, $R$ the gas constant $(8.314 \mathrm{~J} / \mathrm{mol} \mathrm{K})$ and $T$ the absolute temperature (K). Temkin isotherm constants were determined from the plots of $q_{e}$ versus $\ln C_{e}$ as shown in Figure 7. The isotherm constants $A$ and $B$ together with the correlation coefficient, $R^{2}$ value for Temkin isotherm studied are listed in Table 1.

As seen in Table 1, the Freundlich isotherm fits quite well with the experimental data $\left(R^{2}>0.99\right)$, whereas the low correlation coefficients $\left(R^{2}<0.90\right)$ shows poor agreement of Langmuir and Temkin isotherms with the experimental data. The monolayer adsorption capacity according to this model was $166.67 \mathrm{mg} / \mathrm{g}$.

\subsection{Kinetic Studies}

Three kinetic models were applied to adsorption kinetic data in order to investigate the behaviour of adsorption mechanism of AO51 dye onto POEFB. These models are the pseudo-first-order, pseudo-second-order and intra-particle diffusion kinetic models.

Linear form of pseudo-first-order model was described by Lagergren as:

$$
\log \left(q_{e}-q_{t}\right)=\log q_{e}-\frac{k_{1}}{2.303} t
$$

where $q_{e}(\mathrm{mg} / \mathrm{g})$ and $q_{t}(\mathrm{mg} / \mathrm{g})$ are the amount of dye adsorbed at equilibrium and at time $t$, respectively, and $k_{1}$ is the rate constant. Figure 8 shows the plots of $\log \left(q_{e}-q_{t}\right)$ versus $t$ for the pseudo-first-order adsorption kinetics of AO51 onto POEFB. The values of the pseudo-first-order

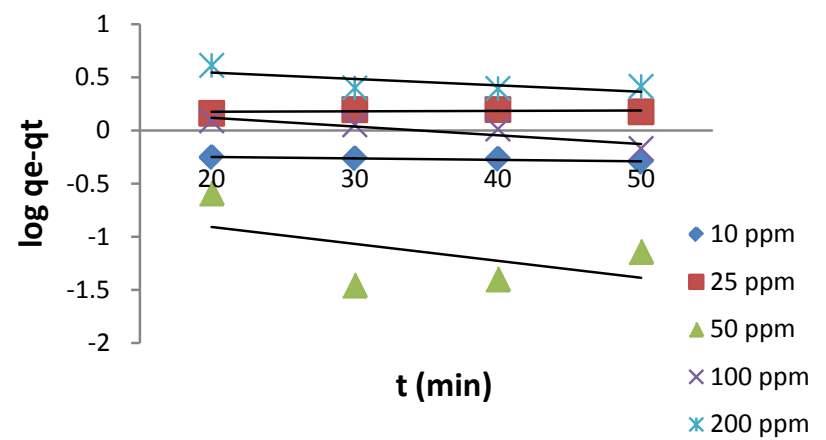

Fig. 8 Pseudo-first-order kinetic for adsorption of AO51 on POEFB

rate constant $k_{1}$ were obtained from the slopes of the straight lines. The $k_{1}$ values, the correlation coefficients, $R^{2}$, and the predicted and experimental $q_{e}$ values are given in Table 2 .

The pseudo-second-order model may be expressed in a linear form as [22]:

$$
\frac{t}{q_{t}}=\frac{1}{k_{2} q_{e}^{2}}+\frac{1}{q_{e}} t
$$

where the equilibrium adsorption capacity, $q_{e}$, and the second-order constant $k_{2}$, can be determined experimentally from the slope and intercept of plot $t / q_{t}$ versus $t$ (Figure 9). The pseudo-second-order rate constants $k_{2}$, the calculated $q_{e}$ values and the corresponding linear regression correlation coefficients values $R^{2}$ are given in Table 2 .

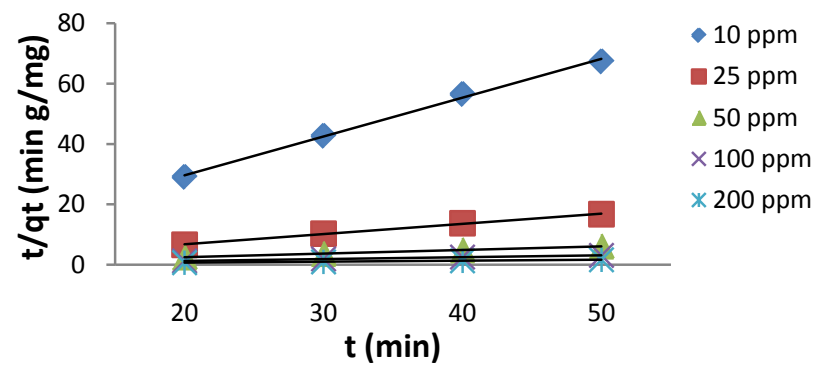

Fig. 9 Pseudo-second-order kinetic for adsorption of AO51 on POEFB

Intraparticle diffusion model was tested to identify the diffusion mechanism of acid orange 51 dyes onto the palm oil fibre. The possibility of intraparticle diffusion was explored by using the intraparticle diffusion model:

$$
q_{t}=k_{\text {diff }} \sqrt{t}+c
$$

where $q_{t}, C$ and $k_{\text {diff }}$ are the amount of dye adsorbed at time $t$, the intercept and the intraparticle diffusion rate constant, 
Table 2 Comparison of the pseudo-first-order, pseudo-second order and particle diffusion rate constants and calculated and experimental $q_{e}$ values obtained at different initial AO51 concentrations

\begin{tabular}{|c|c|c|c|c|c|c|c|c|c|c|}
\hline \multirow{2}{*}{$\begin{array}{l}C_{0} \\
(\mathrm{mg} / \mathrm{l})\end{array}$} & \multirow{2}{*}{$\begin{array}{l}q_{e, e x p} \\
\text { (mg/g) }\end{array}$} & \multicolumn{3}{|c|}{$\begin{array}{c}\text { Pseudo-first-order kinetic } \\
\text { model }\end{array}$} & \multicolumn{3}{|c|}{$\begin{array}{c}\text { Pseudo-second-order kinetic } \\
\text { model }\end{array}$} & \multicolumn{3}{|c|}{ Intraparticle diffusion model } \\
\hline & & $\begin{array}{c}q_{e, c a l} \\
(\mathrm{mg} / \mathrm{g})\end{array}$ & $\begin{array}{c}k_{1} \\
\left(\min ^{-1}\right)\end{array}$ & $R^{2}$ & $\begin{array}{c}q_{e, c a l} \\
(\mathrm{mg} / \mathrm{g})\end{array}$ & $\begin{array}{c}k_{2} \\
\text { (g/mg min) }\end{array}$ & $R^{2}$ & $\begin{array}{c}k_{\text {diff }} \\
\left(\mathrm{mg} / \mathrm{g} \operatorname{~in}^{1 / 2}\right)\end{array}$ & $\begin{array}{c}C \\
(\mathrm{mg} / \mathrm{g})\end{array}$ & $R^{2}$ \\
\hline 10 & 1.25 & 0.58 & 0.03 & 0.88 & 0.08 & 9.32 & 0.99 & 0.01 & 0.75 & 0.11 \\
\hline 25 & 4.49 & 1.49 & 0.01 & 0.10 & 0.30 & 3.29 & 0.99 & 0.02 & 2.93 & 0.24 \\
\hline 50 & 8.23 & 0.18 & 0.37 & 0.27 & 0.83 & 1.14 & 0.99 & 0.02 & 8.01 & 0.36 \\
\hline 100 & 16.82 & 1.59 & 0.19 & 0.84 & 1.65 & 0.53 & 0.99 & 0.14 & 15.05 & 0.91 \\
\hline 200 & 32.64 & 4.03 & 0.14 & 0.55 & 3.11 & 0.28 & 0.99 & 0.43 & 27.23 & 0.94 \\
\hline
\end{tabular}

respectively. Figure 10 show that the plot of $q_{t}$ versus $t^{1 / 2}$ gives a straight line for the intraparticle diffusion for adsorption of AO51 onto POEFB. The intraparticle diffusion rate constants, $k_{\text {diff, and correlation coefficient } R^{2}}$ value are presented in Table 2. As can be seen in Figure 10, the linear line did not pass through the origin and this deviation from the origin or near saturation might be due tothe difference in the mass transfer rate in the initial and final stages of adsorption [13].

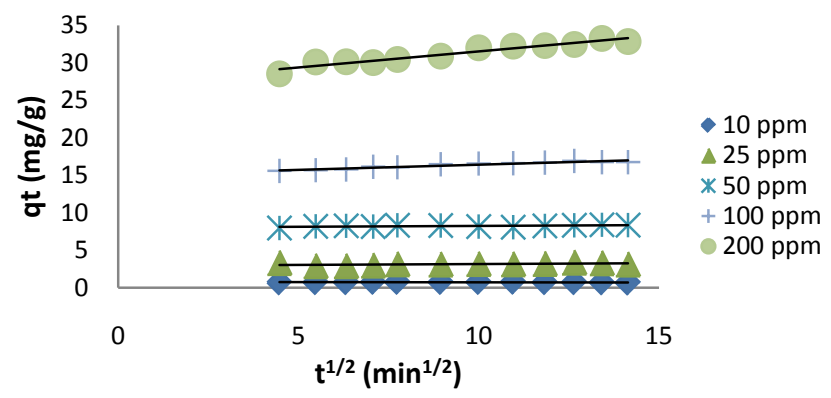

Fig. 10 Intraparticle diffusion model for adsorption of AO51 on POEFB

Table 2 summarizes all the constants and correlation coefficients, $R^{2}$, of the three kinetic models used. For all the systems studied, good correlation coefficients are obtained $\left(R^{2} \approx 1\right)$ by fitting the experimental data to pseudo-secondorder kinetics rather than that of pseudo-first-order and intraparticle diffusion model. This shows that the mechanism of the adsorption of AO51 dye by POEFB can be described by the pseudo-second-order kinetic model, based on the assumption that the rate-limiting step may be chemisorptions involving valency forces through sharing or exchange of electrons between sorbent and sorbate [22]. Based on the Table 2, the value of $q_{e}$ increased as the initial concentration of the adsorbate increased. The value of the overall rate constants also varies as the initial concentration varied. Similar phenomenan has been observed in the adsorption of acid dyes by activated palm ash [19], ground eggshell waste [23], activated bleaching earth [24] and mixed sorbents [25].

\section{CONCLUSION}

Palm oil empty fruit bunch, an agricultural solid waste, was successfully utilized as a low-cost alternative adsorbent for the removal of hazardous dye like acid orange 51. Since POEFB in this work is freely, abundantly and locally available, the resulting sorbent is expected to be economically viable for removal acid dye from aqueous solution. The equilibrium adsorption data were analyzed by the Langmuir isotherm, Freundlich isotherm and Temkin isotherm models. The result indicated that the equilibrium sorption fitted well with the Freundlich isotherm, displaying higher regression coefficient, $R^{2}$ value. The monolayer adsorption capacity of POEFB was found to be 166.67 $\mathrm{mg} / \mathrm{g}$. The adsorption kinetics can be well described by the pseudo-second-order model equation.

\section{ACKNOWLEDGEMENT}

The author thanks the Department of Chemistry, Faculty of Science, Universiti Teknologi Malaysia, Johor for facilities as well as to Universiti Malaysia Sabah for funding the project.

\section{REFERENCES}

[1] C. Parvathi and T. Maruthavanan, Indian J. Sci. Tech., 3(4) (2010) 408-410.

[2] F.A.Pavan, E.C. Lima, S.L.P. Dias and A.C.Mazzocato, J. Hazard. Materials, 150(2007) 703-712.

[3] M.Rafatullah, O.Sulaiman, R.Hashim and A. Ahmad, J. Hazard. Materials,Article in Press, 2009.

[4] E.Forgacsa, T.Cserhatia, and G.Oros, Environ. Int., 30 (2004) 953971.

[5] M. J.Martin, A.Artola, M. D.Balaguer and M. Rigola, Chem. Eng. J., 94(2003) 231-239.

[6] S. Venkata Mohan, P. Sailaja, M. Srimurali, J. Karthikeyan, Environ. Engg.\& Policy 1, 6(1999) 149-154.

[7] Y.C. Wong, Y.S. Szeto, W.H. Cheung and G. Mckay, Process Biochemistry, 39(2004)693-702.

[8] N. Rajamohan, African J. of Environ. Sci\& Tech. Vol 3, 11(2009) 399-404.

[9] H.M.Chiang, T.C.Chen, S.D.Pan and H.L.Chiang, J.ofHazardous Materials, 161 (2009) 1384-1390. 
[10] R. Cheng, S. Ou, B. Xiang, Y. Li and Q. Liao, Langmuir, 26(2)(2010)752-758.

[11] H.P.S.A.Khalil, M.S.Alwani, R.Ridzuan, H. Kamarudinand A.Khairul,Polymer-Plastics Tech. and Eng. 47(2008) 273-280.

[12] K.Y.Foo and B.H.Hameed, J. Hazard. Materials, 172(2009) 523531.

[13] I.A.W.Tan, B.H.Hameed, and A.L.Ahmad,Chem. Eng. J., 127 (2007) 111-119.

[14] A.E.Ofomaja and Y.S.Ho,Dyes and Pigments, 74 (2007) 60-66

[15] B.H.Hameed and M.I.El-Khaiary, J. of Hazard. Mat., 154(2008) 237-244.

[16] S.A.Saad, S.Daud, F.H.Kasim and M.N.Saleh,Proceeding of the International Conference of Sustainable Materials (ICoSM 2007), Universiti Malaysia Perlis, Malaysia, (2007) 297-299.

[17] S.A.Saad, S.Daud, F.H.Kasim and M.N.Saleh, Proceeding of the International Conference of Sustainable Materials (ICoSM 2007), Universiti Malaysia Perlis, Malaysia, (2007) 293-296.
[18] L.S.Tan, K.Jain and C.A.Rozaini, J. App. Sci. Env. Sanitary, 5 (2010) 266-276.

[19] B.H.Hameed, A.A.Ahmad and N.Aziz, Chem. Eng. J., 133(2007) 195-203.

[20] B.H.Hameed and M.I.El-Khaiary, J. of Hazard. Mat., 154 (2008) 237-244.

[21] Y.S.Ho and G.Mckay, Process Biochem., 34 (1999) 451-465

[22] S.T.Ong, W.N.Lee, P.S.Keng, S.L.Lee, Y.T.Hung, and Int. J. Phys. Sci., 5(5) 582-595.

[23] W.T.Tsai, K.J.Hsien, H.C.Hsu, C.M.Lin, K.Y.Lin and C.H.Chiu,BioresourceTechn., 99 (2008) 1623-1629

[24] W.T.Tsai, C.Y.Chang, C.H.Ing and C.F.Chang, J. Colloids and Interface Sci., 275(2004) 72-78.

[25] Y.S.Ho and C.C.Chiang, Adsorption, 7 (2001) 139-147. 